Tecno Lógicas

ISSN 0123-7799

Vol. 18, No. 34, pp. 87-99

Enero-junio de 2015

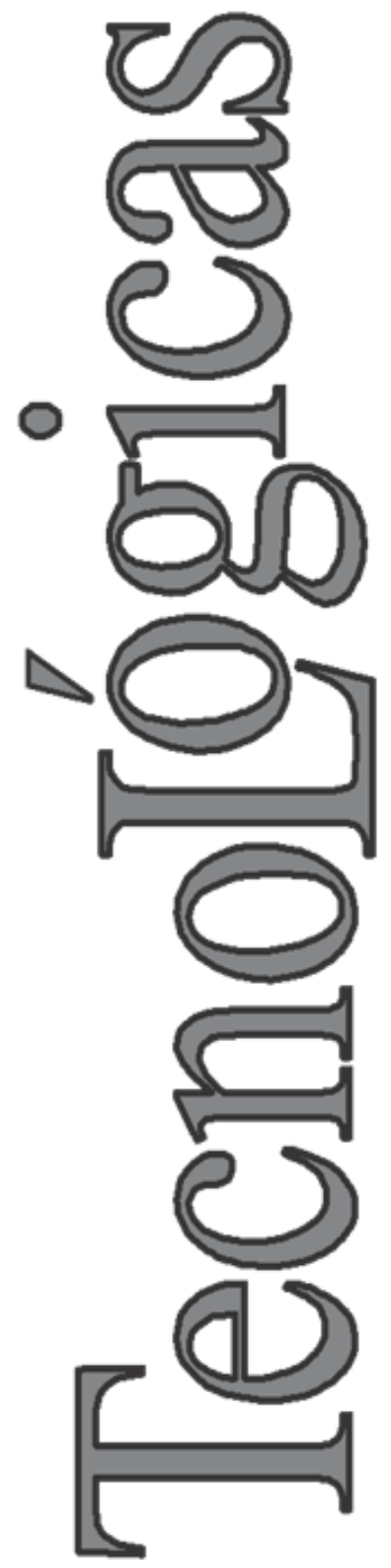

(C) Copyright 2015 por autores y Tecno Lógicas Este trabajo está licenciado bajo una Licencia Internacional Creative Commons Atribución (CC BY)

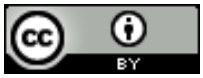

\section{Desempeño de redes inalámbricas y redes industriales inalámbricas en procesos de control en tiempo real bajo ambientes industriales}

\section{Performance in wireless networks and industrial wireless networks on control processes in real time under industrial environments}

\author{
Juan F. Monsalve-Posada ${ }^{1}$, Alexander Arias-Londoño ${ }^{2}$ \\ y Juan G. Mejía-Arango ${ }^{3}$
}

Recibido: 26 de junio de 2014, Aceptado: 1 de diciembre de 2014

Cómo citar / How to cite

J. F. Monsalve-Posada, A. Arias-Londoño y J. G. Mejía-Arango, "Desempeño de redes inalámbricas y redes industriales inalámbricas en procesos de control en tiempo real bajo ambientes industriales", Tecno Lógicas, vol. 18, no. 34, pp. 87-99, 2015.

1 Ingeniero electrónico, Departamento de Electrónica y Telecomunicaciones, Instituto Tecnológico Metropolitano, MedellínColombia, jfmposada@gmail.com

2 Magíster en Ciencias Computacionales, Departamento de Electrónica y Telecomunicaciones, Instituto Tecnológico Metropolitano, Medellín-Colombia, profeitm@gmail.com

3 Magíster en Gestión Energética Industrial, Departamento de Electrónica y Telecomunicaciones, Instituto Tecnológico Metropolitano, Medellín-Colombia, juanmejia@itm.edu.co 


\section{Resumen}

El uso creciente de las redes Ethernet en el piso de la pirámide de automatización ha hecho que muchas compañías desarrollen dispositivos para operar bajo los requerimientos característicos de este nivel, en la actualidad denominada red Industrial Ethernet. A nivel industrial se encuentra en el mercado variedad de sensores y actuadores que incorporan esta tecnología, muchos de estos equipos se venden a muy alto costo, por eso en este artículo se evaluó el desempeño de dos redes inalámbricas, una con dispositivos del Ethernet convencional, y otra con dispositivos de Industrial Ethernet variando cuatro parámetros que son: distancia, número de bytes, relación señal a ruido y la tasa de error de paquetes, midiendo los retardos y comparando con métricas estadísticas los resultados, para ello se usaron gráficas Box Plot. Al final, se concluye que bajo los parámetros y condiciones evaluados, las redes inalámbricas pueden servir como sistema de comunicación en aplicaciones de control con retardos permisibles de hasta 50 ms, además, los resultados demuestran un mejor desempeño de las redes Industrial Ethernet con respecto a las convencionales, con diferencias en los RTT de milisegundos. Por lo tanto, se recomienda establecer qué riesgo representa para el proceso a controlar estos retardos y así determinar si los equipos convencionales aplican, ya que bajo ciertas características, como condiciones de humedad y temperatura, pueden operar adecuadamente durante un tiempo considerable y a un menor costo que los dispositivos de Industrial Ethernet.

\section{Palabras clave}

Industrial Ethernet, IWLAN, control, ambientes industriales, tiempo real.

\section{Abstract}

The growing use of Ethernet networks on the industrial automation pyramid has led many companies to develop new devices to operate in requirements of this level, nowadays it is called Industrial Ethernet network, on the market there are various sensors and actuators to industrial scale equipped with this technology, many of these devices are very expensive. In this paper, the performance of two wireless networks is evaluated, the first network has conventional Ethernet devices, and the second network has Industrial Ethernet devices. For the process we vary four parameters such as distance, number of bytes, the signal to noise ratio, and the packet error rate, and then we measure delays and compare with metric statistics results, Box Plot graphs were used for the analysis. Finally, we conclude that under the parameters and conditions tested, wireless networks can serve as a communication system in control applications with allowable delays of up to $50 \mathrm{~ms}$, in addition, the results show a better performance of Industrial Ethernet networks over conventional networks, with differences in the RTT of milliseconds. Therefore, it is recommended to establish what risk is for the process to control these delays to determine if the equipment conventional applies, since under certain features like humidity and temperature can operate properly for a considerable time and at lower cost than devices to Industrial Ethernet.

\section{Keywords}

Industrial Ethernet, IWLAN, Control, Industrial Environment, Real Time. 


\section{INTRODUCCIÓN}

En la actualidad, los sistemas de control afrontan cambios y retos ocasionados por varios motivos, entre ellos la complejidad en los procesos de control en busca de una mejor calidad a menor precio en la producción, y otra no tan evidente pero que ha sido muy importante, la seguridad de los procesos productivos de tal forma que no represente riesgos para los operarios presentes en dichos procesos [1], [2]. En este contexto y de cierta forma buscando lograr estos objetivos, las redes inalámbricas han atraído la atención en los procesos de control, de manera que la industria de la automatización ha centrado sus mayores esfuerzos en el desarrollo de nuevos protocolos [3].

Hay un gran interés en la investigación y el desarrollo de redes inalámbricas [4]. En los sistemas de control en red, por ejemplo, hay un gran interés en el desarrollo de la tecnología inalámbrica como un reemplazo potencial para la actual generación de redes cableadas industriales [5]. Estas redes cableadas presentan grandes inconvenientes y desventajas que pretenden ser solucionadas mediante el uso de otras tecnologías como las comunicaciones inalámbricas. Las tecnologías cableadas actuales presentan dificultades en ciertos ambientes como la industria petroquímica y donde se usen dispositivos móviles y portátiles [6], [7].

Aplicaciones en lo militar, la industria, medicina y sectores comerciales son las que más motivan la solución de problemas cruciales asociados con los retardos y la pérdida de datos comunes en redes inalámbricas. La última meta aquí es aplicar redes inalámbricas para lograr control realimentado en tiempo real comparable al control cableado punto a punto [8]. Los dos principales problemas de las redes inalámbricas utilizadas para control en ambientes industriales son, la poca fiabilidad del medio de trasmisión causada por el comportamiento no determinístico especial- mente en IEEE802.11 que utiliza CSMA/CA como protocolo de acceso al medio, y los altos retardos causados por interferencia y la trayectoria multicaminos [9].

A pesar de los problemas que poseen las redes inalámbricas $\mathrm{WiFi}$, en la actualidad se sigue investigando para aplicaciones industriales por tres razones fundamentales. La primera es que aunque IEEE802.15 ofrece mejores técnicas en ahorro de energía y mejores resultados en cuanto a fiabilidad, es menos robusto a la interferencia y al ruido que el IEEE802.11 [10]. Segundo, aunque hay aplicaciones industriales con menos de 10 nodos trasmitiendo datos por debajo de los 80 bytes, también hay aplicaciones que utilizan cientos de nodos transmitiendo por encima de 80 bytes, para estas el IEEE802.15.4 presenta problemas [11]. Y tercero es el posicionamiento en el mercado de dispositivos inalámbricos diseñados para ambientes industriales como los SCALANCE W744-1Pro y W788-1Pro de Siemens, ambos trabajando con el protocolo IEEE802.11.

De igual forma, existe la necesidad de caracterizar y evaluar el comportamiento de estos dispositivos inalámbricos diseñados especialmente para ambientes industriales [12], esto permite determinar si se cumple con los estándares especificados por el fabricante. Un objetivo de Industrial Ethernet es extender sus aplicaciones hasta la base de la pirámide de automatización para comunicar procesos de control en tiempo real bajo condiciones extremas teniendo en cuenta que, dependiendo de la aplicación, la mayoría de los procesos de control industrial pueden tolerar latencias entre 10 - $50 \mathrm{~ms}$ [13].

En este artículo se hace una comparación experimental entre el desempeño de una red inalámbrica y una red industrial inalámbrica para procesos de control en tiempo real bajo ambientes industriales, evaluando 4 parámetros que son: distancia, tamaño de datos, relación señal a ruido (SNR) y PER (Packet Error Rate). El artículo se presenta de la siguiente forma: en 
la sección dos se presenta la metodología, en la sección tres los resultados y discusiones, y en la sección cuatro las conclusiones.

\section{METOdOLOGíA}

En la Fig. 1 se muestra el esquema propuesto para evaluar el desempeño de un sistema de comunicación con industrial Ethernet bajo ambientes industriales. Para la comunicación con Ethernet convencional basta sustituir los dispositivos empleados en industrial Ethernet, incluyendo los cables usados para comunicar los puntos de acceso (AP) con el switch.

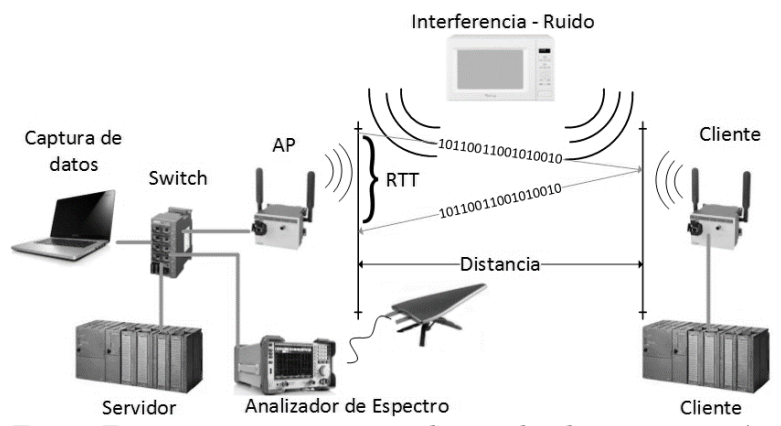

Fig. 1. Esquema propuesto para la prueba de comunicación Industrial Ethernet inalámbrica. Fuente: Autores

En este artículo se pondrá a prueba la comunicación variando tres parámetros que afectan considerablemente su desempeño.

\subsection{Relación señal a ruido SNR}

El SNR se define como (1):

$\operatorname{SNR}(d B)=10 \log P_{S}-10 \log P_{n}$

En la cual $P_{s}$ es la potencia de la señal (Watts) y $P_{n}$ la potencia del ruido (Watts). La SNR se debe tener en cuenta a la hora de establecer una comunicación inalámbrica ya que afecta considerablemente su desempeño, sobre todo en ambientes industriales donde el nivel de potencia del ruido es grande. Para medir el SNR se usó el analizador de espectro Rohde \& Schwarz
FSH3 que tiene un ancho de banda de 3 $\mathrm{GHz}$ y como transductor una antena directiva de banda ancha HyperLOG 7060 (700 $\mathrm{MHz}-6 \mathrm{GHz}$ ) con ganancia de $5 \mathrm{dBi}$. Para adaptar el transductor al analizador se editó la librería de esta con la tabla de datos del factor de antena suministrados por el fabricante. Este factor tiene unidades de $\mu \mathrm{V} / \mathrm{m}$, por lo tanto todas las medidas realizadas con el analizador fueron en unidades de campo eléctrico. En la Fig. 2a se muestra un ejemplo de las mediciones hechas para IEEE $802.11 \mathrm{~g}$ sin el ruido introducido con un microondas, la Fig. $2 \mathrm{~b}$ muestra cómo el nivel del ruido se incrementa con el horno microondas.

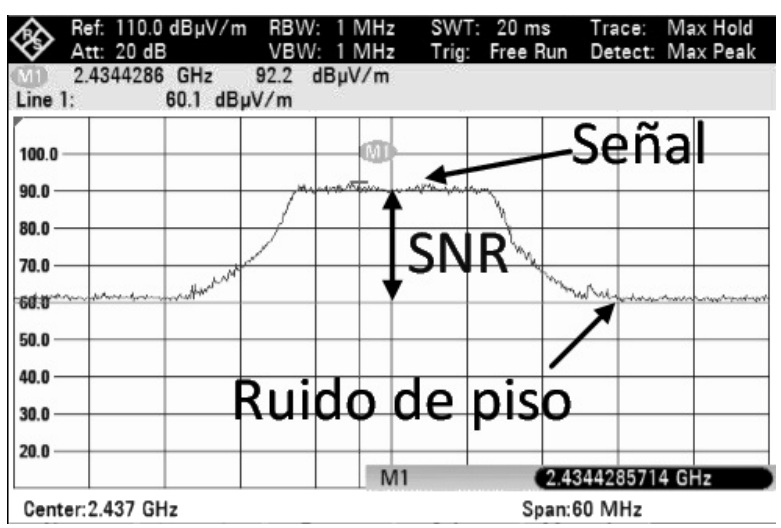

a)

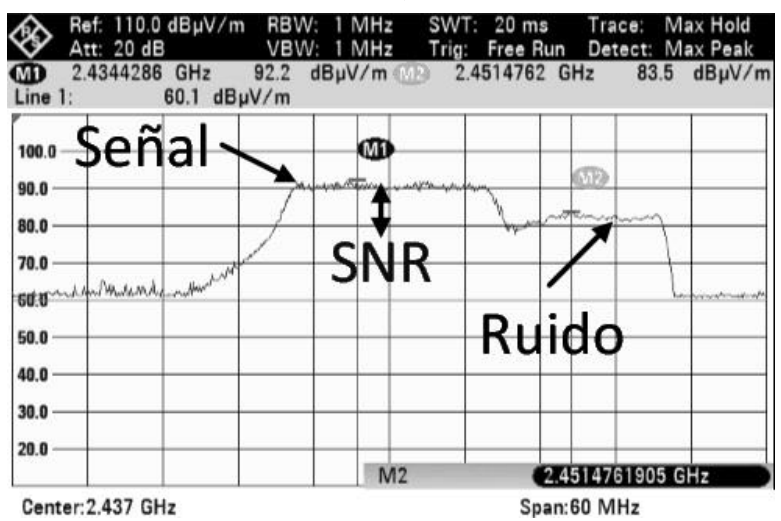

b)

Fig. 2. Medidas de SNR para $2437 \mathrm{MHz}$ correspondiente al canal 6 de IEEE 802.11g. Fuente: Autores

El nivel de campo eléctrico de la señal se toma como el pico máximo identificado por el marcador M1, y el nivel de campo eléctrico del ruido se tomó como valor mí- 
nimo o ruido de piso identificado por la línea 1 o el marcador M2.

Para variar el SNR en el experimento y emular los ambientes industriales, se usa un horno microondas que fue implementado como una gran fuente de perturbación para redes inalámbricas [14]. En una prueba inicial, se dejó encendido el microondas con una carga de agua durante 3 minutos, la intensidad de campo, el ancho de banda y la frecuencia central del ruido emitido fue de $-57,5 \mathrm{dBm}, 60 \mathrm{MHz}$ y 2439 $\mathrm{MHz}$, respectivamente. Para el experimento con el radio enlace funcionando se tomaron tres medidas; la primera con el microondas apagado; la segunda con el microondas encendido durante 3 minutos y a una distancia de $100 \mathrm{~cm}$ del servidor; y la tercera, igual que la anterior, pero con una distancia al servidor de $20 \mathrm{~cm}$. Con esto se obtuvieron SNR promedio de 29,54 dBm, $10,985 \mathrm{dBm}$ y $0,66 \mathrm{dBm}$, respectivamente, con un número total de 108 mediciones.

\subsection{Distancia}

La distancia está directamente relacionada con el desvanecimiento de la señal y los problemas por trayectoria multicamino que en definitiva afectan la calidad de la transmisión de acuerdo con (2) y (3):

$$
\begin{aligned}
& L_{p(d B)}=20\left(\log \frac{4 \pi}{c}+\log f+\log D\right) \\
& F_{m}=30 \log D+ 10 \log (6 A B f) \\
&- 10 \log (1-R)-70
\end{aligned}
$$

Donde $L_{p(d B)}$ son las pérdidas en la trayectoria en espacio libre, $F_{m(d B)}$ es el margen de desvanecimiento, $c$ la velocidad de la luz en el vacío, $D$ la distancia en metros, $f$ la frecuencia en Hertz, $A$ el factor de aspereza del terreno, $B$ el factor climático y $R$ la confiabilidad. Para el experimento se hicieron pruebas para distancias de $2,10 \mathrm{y}$ 30 metros, teniendo en cuenta las recomendaciones de Industrial Ethernet de poner lo más cerca posible los dispositivos de la red. En la Tabla 1 se muestran las $L_{p(d B)}$ y el $F_{m(d B)}$ para las distancias tomadas, donde se observa cómo las pérdidas aumentan con la distancia.

\begin{tabular}{ccccc}
\multicolumn{5}{c}{ Tabla 1. Pérdidas por trayectoria para IEEE $802.11 \mathrm{~b} / \mathrm{g} \mathrm{y}$} \\
a/h. Fuente: Autores \\
\hline Estándar & Frecuencia & Distancia & $L_{p(d B)}$ & $F_{m(d B)}$ \\
\hline IEEE & & $2 \mathrm{~m}$ & 46,20 & 73,70 \\
802.11 & $2437 \mathrm{MHz}$ & $10 \mathrm{~m}$ & 60,20 & 94,70 \\
$\mathrm{~b} / \mathrm{g}$ & $(\mathrm{Ch} 6)$ & $30 \mathrm{~m}$ & 69,72 & 109,00 \\
& & $2 \mathrm{~m}$ & 52,75 & 76,97 \\
IEEE & $5180 \mathrm{MHz}$ & $10 \mathrm{~m}$ & 66,73 & 97,94 \\
802.11 & $(\mathrm{Ch} 36)$ & $30 \mathrm{~m}$ & 72,27 & 112,20 \\
a/h & & & &
\end{tabular}

\subsection{Número de bytes}

Otro parámetro a evaluar será el número de bytes de datos enviados del PLC servidor al PLC cliente. Se debe tener en cuenta que en el piso de la pirámide de automatización, el número de bytes de datos es pequeño y dependiendo del número de dispositivos conectados, el flujo de información puede ser alto. Para la prueba se enviaron paquetes de datos de 8, 32 y 64 bytes. Otro parámetro que está relacionado con el número de bytes y que se usó para el análisis de resultados es el PER que está relacionado con la interferencia, la potencia de transmisión, el tipo de modulación y el ancho de banda [15], se define con (4):

$p_{k}=\left(\begin{array}{l}n \\ k\end{array}\right) p^{k}(1-p)^{n-k}$

Donde $p$ es la probabilidad de error, $n$ el número total de paquetes y $p_{k}$ la probabilidad de error de $k$ paquetes. La Tabla 2 muestra las estadísticas de tráfico para la comunicación Ethernet convencional tomada del AP D-Link, en este caso, el PER se encuentra con (5):

$P E R=\frac{\text { Received Frame FCS Error Count }}{\text { Received Frame Count }} \times 100$

En la Tabla 3 están las estadísticas de tráfico obtenidas de Industrial Ethernet tomadas del AP Scalance W788-1 Pro, el PER se encuentra con (6): 
Desempeño de redes inalámbricas y redes industriales inalámbricas en procesos de control en tiempo real...

$$
P E R=\frac{\text { Receiving errors }}{(\text { Received Frame Count })} \times 100
$$

Tabla 2. Estadísticas de tráfico tomadas para Ethernet convencional. Fuente: Autores

\begin{tabular}{lc}
\hline \multicolumn{2}{c}{ Throughput } \\
Transmit success rate & $99 \%$ \\
Transmit retry rate & $8 \%$ \\
Receive success rate & $78 \%$ \\
Receive duplicate rate & $1 \%$ \\
RTS success count & 0 \\
RTS failure count & 14175 \\
Transmitted bytes count & 2845429 \\
Received bytes count & 5671036 \\
\hline \multicolumn{2}{c}{ Transmitted frame count } \\
Transmitted frame count \\
Multicast transmitted frame count \\
Transmitted error count \\
Transmitted total retry count \\
Transmitted multiple total retry count \\
\multicolumn{2}{c}{ Received frame count } \\
\hline \multicolumn{2}{c}{ WEP frame error count } \\
Received frame count \\
Multicast received frame count \\
Received frame FCS error count \\
Received frame duplicate count \\
ACK RCV failure count \\
\hline \multicolumn{2}{c}{ W } \\
WEP excluded frame count \\
WEP ICV error count \\
\hline
\end{tabular}

Tabla 3. Estadísticas de tráfico tomadas para Industrial Ethernet. Fuente: Autores

\begin{tabular}{lcc}
\hline \multicolumn{3}{c}{ Traffic statistics } \\
\cline { 2 - 3 } Rate & Receive & Transmit \\
\cline { 2 - 3 } Signal strength & 11,0 Mbits & 11,0 Mbits \\
Frame count & $100 \%$ & N/A \\
Bytes count & 81585 & 87591 \\
Data frame count & 52196444 & 7382237 \\
Data bytes count & 81586 & 86972 \\
Unicast & 53666506 & 7283396 \\
Multicast & 81581 & 67811 \\
Broadcast & 4 & 118 \\
Control framers & 5 & 80 \\
Management frames & 0 & 0 \\
\hline & 2271 & 739 \\
\hline Receiving errors & & \\
Transmission errors & & 1530 \\
Discarded frames & & 52559 \\
Dropped frames & & 2 \\
Acknowledge errors & & 75 \\
Filtered frames & & 65100 \\
Duplicate frames & & 49717 \\
RTS errors & & 2 \\
CRC errors & & 3348 \\
Transmission retries & & 1530 \\
Encryption errors & & 27728 \\
\hline
\end{tabular}

\subsection{RT (Roud Trip delay Time)}

Para el control en tiempo real es fundamental conocer el retardo ocasionado por el medio de trasmisión, ya que a partir de este se puede determinar, de acuerdo con la planta, si permite obtener la respuesta deseada establecida por el controlador, además se debe tener en cuenta en el diseño de este. Es por esto que una de las métricas más relevantes para medir el desempeño de una red utilizada para control en tiempo real es el retraso que sufren los datos. El término relacionado para esta medida es el retardo RTT (tiempo de ida y retorno) que es definido como el tiempo que transcurre mientras se envía un paquete y se recibe el reconocimiento (ACK), y es usado para establecer la velocidad de conexión en tiempo real entre dos hosts [16]. En la Fig. 3 se presenta el algoritmo implementado en los PLC's para medir el retardo al variar el número $(\mathrm{N})$ de bytes de datos.

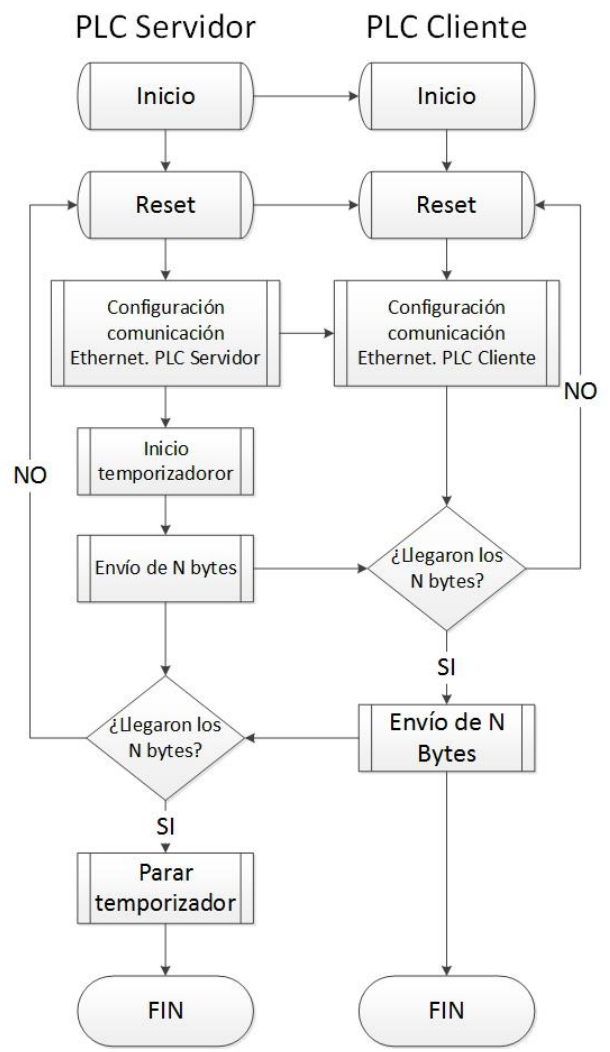

Fig. 3. Algoritmo implementado para medir el RTT entre dos PLC's. Fuente: Autores 


\subsection{Pruebas en un proceso de control en tiempo real}

Por último, para evaluar el desempeño de los dos sistemas de comunicación en un proceso de control en tiempo real, se usó el experimento que se muestra en la Fig. 4.

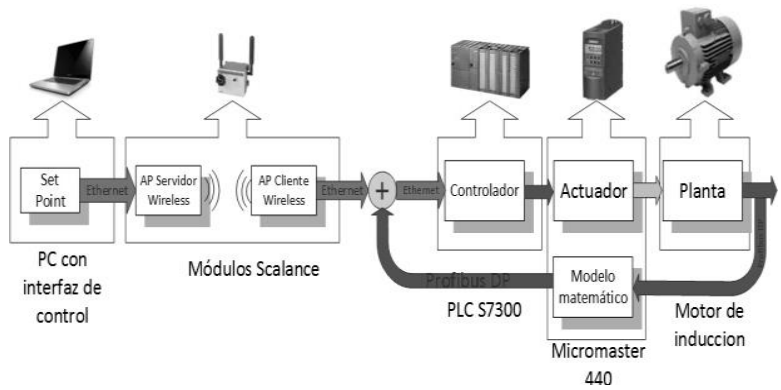

Fig. 4. Escenario para las pruebas en un proceso de control en tiempo real. Fuente: Autores

En el PC se diseñó una interfaz de control que cuenta con 4 características (ver Fig. 5); la primera para cargar los parámetros del motor en el variador (Micromaster 440); la segunda para guardar los datos obtenidos en un archivo de texto; la tercera para enviar parámetros de control al motor; y la cuarta para mostrar las gráficas de respuesta del motor. Para la prueba se envía por red inalámbrica el valor deseado en el motor y se lee la respuesta de este para medir el tiempo que tarda el motor en llegar al valor deseado variando el SNR.

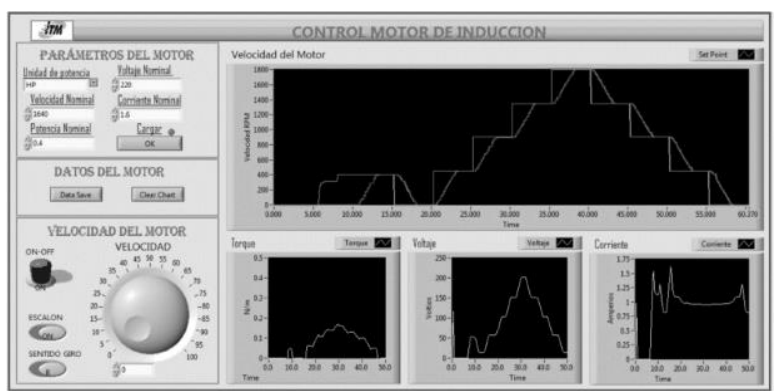

Fig. 5. Aplicación en LabView para analizar el desempeño de la comunicación inalámbrica en un proceso de control en tiempo real. Fuente: Autores

\subsection{Características de los dispositivos em- pleados en el experimento}

De los equipos usados, los que determinarán sustancialmente el desempeño de la red son los AP's ya que permiten configurar, administrar y supervisar la red. Por esta razón, a continuación se describen las características más importantes de los AP's de Ethernet industrial y convencional, de tal forma que se pueda hacer una comparación sobre algunos parámetros importante en ambiente industriales.

\subsubsection{Scalance W788-1 PRO y W744-1 PRO}

El módulo W788-1 pro actúa como AP y el módulo W744-1 pro se conecta al AP como cliente. Con estos es posible conectar estaciones fijas o móviles con conexión Industrial Ethernet que deban ser integradas en las redes de radiotransmisión Industrial Wireless Lan (IWLAN), con un alto grado de fiabilidad en las comunicaciones [17]. En la Tabla 4 se pueden observar las principales características de estos módulos inalámbricos para Industrial Ethernet.

Tabla 4. Características de los módulos inalámbricos Scalance. Fuente: [17]

\begin{tabular}{|c|c|}
\hline \multicolumn{2}{|c|}{ Scalance W788-1 Pro y W744-1 Pro } \\
\hline $\begin{array}{l}\text { Velocidad de transmisión } \\
\text { inalámbrica }\end{array}$ & $1 \ldots 108 \mathrm{Mbps}$ \\
\hline Estándares inalámbricos & $\begin{array}{c}\text { IEEE } 802.11 \mathrm{a}, 802.11 \mathrm{~h}, \\
802.11 \mathrm{~b}, 802.11 \mathrm{~g} \text { y } 802.11 \mathrm{~g} \\
\text { Turbo }\end{array}$ \\
\hline Bandas de frecuencia & $2,5 \mathrm{GHz}$ y $5 \mathrm{GHz}$ \\
\hline Consumo de potencia & $\leq 9 \mathrm{~W}$ \\
\hline Ganancia a $2,45 / 5,25 \mathrm{GHz}$ & $3 / 5 \mathrm{dBi}$ \\
\hline Sensibilidad de recepción & $-98 \mathrm{a}-74 \mathrm{dBm}$ \\
\hline Grado de protección & IP65 \\
\hline Temperatura de operación & $-20^{\circ} \ldots 60^{\circ} \mathrm{C}$ \\
\hline Humedad no condensada & $95 \%$ \\
\hline
\end{tabular}




\subsubsection{D-LINK DWL-8600 AP}

Este punto de acceso, diseñado para su instalación en interiores, ofrece opciones seguras para que los administradores de red desarrollen una red inalámbrica sumamente robusta y muy gestionable [18]. En la Tabla 5 se especifican las principales características de estos AP.

Tabla 5. Características de los AP D-Link. Fuente: [18]

\begin{tabular}{lc}
\hline \multicolumn{2}{c}{ D-Link DWL-8500AP } \\
\hline $\begin{array}{lc}\text { Velocidad de transmisión } \\
\text { inalámbrica }\end{array}$ & $6 \ldots 108 \mathrm{Mbps}$ \\
Estándares inalámbricos & 802.11b, IEEE 802.11g, \\
& IEEE 802.11a Turbo \\
Bandas de frecuencia & $2,5 \mathrm{GHz}$ y $5 \mathrm{GHz}$ \\
Consumo de potencia & $\leq 9 \mathrm{~W}$ \\
Ganancia a 2.45/5.25 GHz & $2,5 / 5,5 \mathrm{dBi}$ \\
Sensibilidad de recepción & -92 a $-68 \mathrm{dBm}$ \\
Grado de protección & No reporta \\
Temperatura de operación & $0^{\circ} \ldots 40^{\circ} \mathrm{C}$ \\
Humedad no condensada & $90 \%$ \\
\hline
\end{tabular}

\section{RESULTADOS Y DISCUSIÓN}

Los resultados se clasifican de acuerdo con los parámetros variados que son: número de datos, distancia y SNR. Para mostrar los datos obtenidos se usaron gráficas boxplots, ya que muestran variables estadísticas de interés como la media, la dispersión de los datos, los máximos y mínimos. Con esta metodología se tomaron 720 mediciones calculadas así: dos variaciones de SNR $(29,54$ y $0,66 \mathrm{dBm})$, tres variaciones de distancia $(2,10$ y $30 \mathrm{~m})$, tres variaciones en el tamaño de bytes $(8,32$ y 64 bytes), cuatro protocolos (802.11g y gT para ambas redes), y diez medidas por cada variación con lo que se obtuvieron 720 mediciones del RTT.

\subsection{Número de datos}

En la Fig. 6 se presenta el boxplot variando el número de bytes para una $\mathrm{SNR}=29,54 \mathrm{dBm}$ entre la red inalámbrica con elementos de Industrial Ethernet y el convencional. Se observa que si aumenta el tamaño de datos enviados, el RTT aumenta. Además, para $802.11 \mathrm{~g}$ hay una mayor dispersión de retardos que para $802.11 \mathrm{gT}$, en cuanto a Industrial Ethernet. En el Ethernet convencional se puede ver que la diferencia en cuanto a retardos es pequeña, para el primero se tiene un RTT promedio de 14 a $18 \mathrm{~ms}$ y para el segundo desde 14 a $20 \mathrm{~ms}$, ambos en 802.11g.
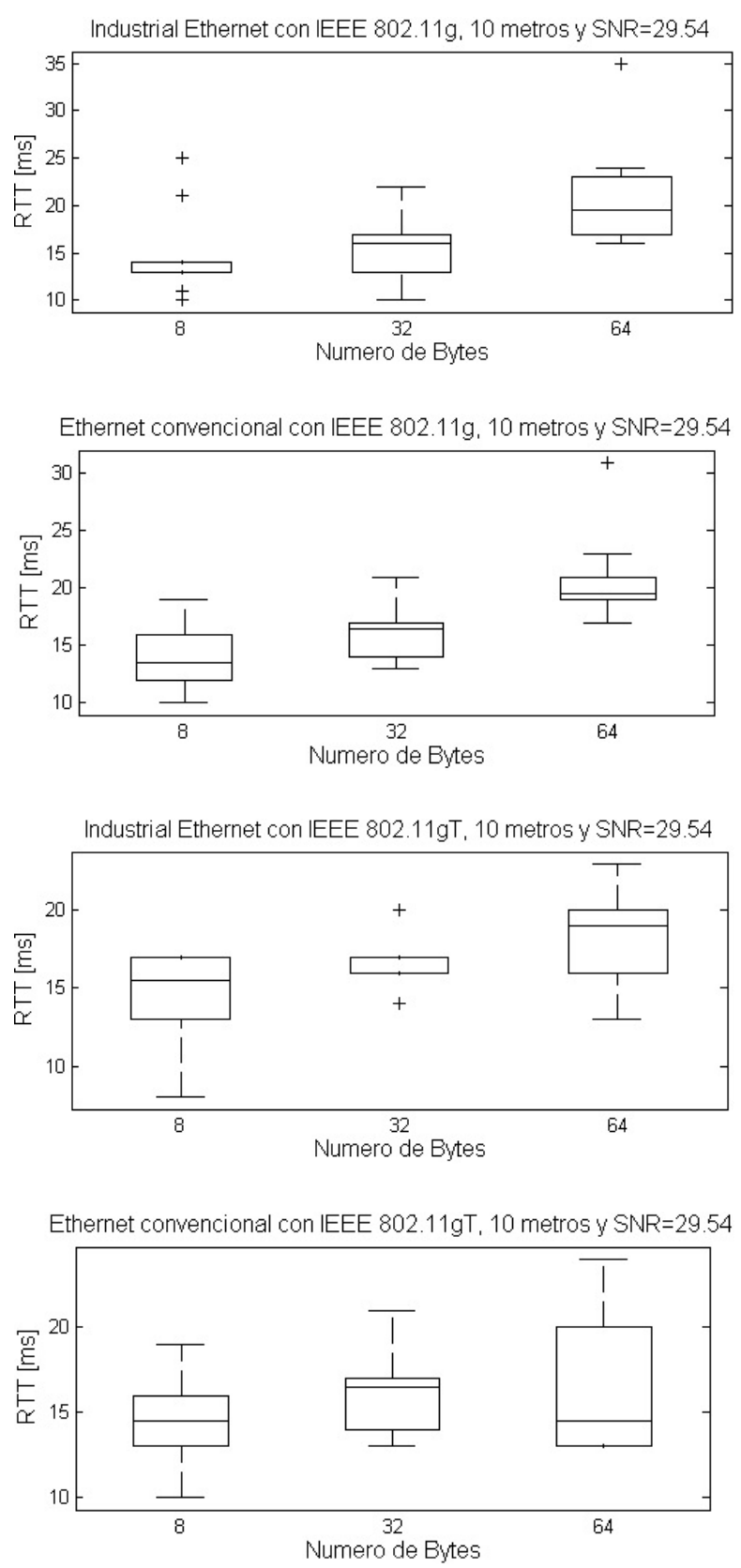

Fig. 6. Resultados obtenidos variando el número de bytes con $\mathrm{SNR}=29,54 \mathrm{dBm}$. Fuente: Autores 
En la Fig. 7 se aprecia que cuando disminuye el SNR, el RTT promedio aumenta. Para Industrial Ethernet los RTT promedio van de 19 a $29 \mathrm{~ms}$ y para Ethernet convencional de 20 a $25 \mathrm{~ms}$. Para $802.11 \mathrm{gT}$ de Industrial Ethernet se observan muy buenos resultados comparado con $802.11 \mathrm{~g}$, el promedio RTT pasa de $19 \mathrm{~ms}$ (64 bytes) sin ruido a $24 \mathrm{~ms}$ ( 64 bytes) con $\mathrm{SNR}=0,66$ dBm y baja dispersión de los cuartiles Q1 a Q3 que conforman el 50\% de los datos.
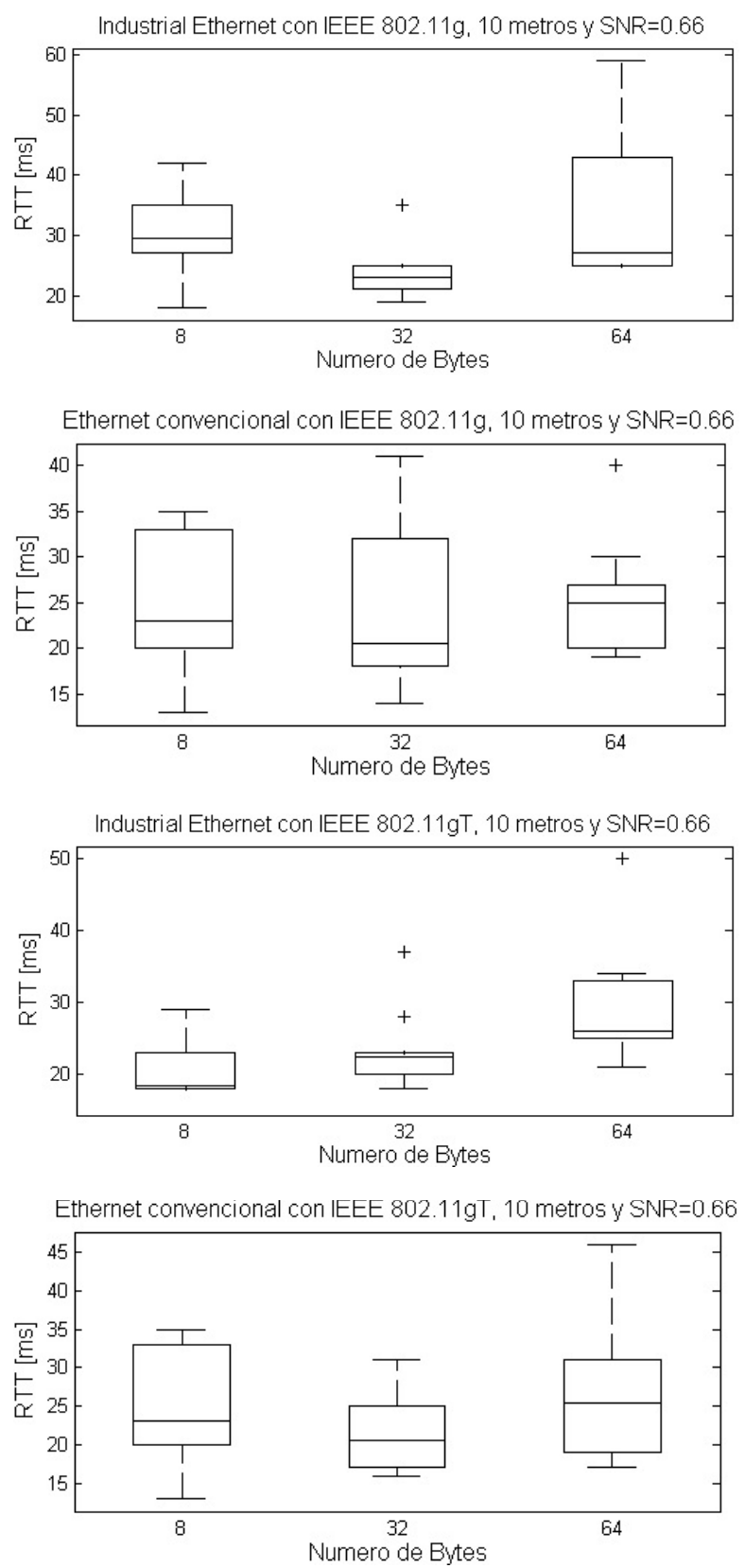

Fig. 7. Resultados obtenidos variando el número de bytes con $\mathrm{SNR}=0,66 \mathrm{dBm}$. Fuente: Autores

\subsection{Distancia}

A continuación se muestran los resultados variando la distancia con $802.11 \mathrm{~g} \mathrm{y}$ gT, 64 bytes de datos y SNR=29,54 dBm. En la Fig. 8 se observa que en la medida que aumenta la distancia, aumenta el RTT. Por ejemplo, para $802.11 \mathrm{~g}$ con Industrial Ethernet, se pasa de $18 \mathrm{~ms}(2 \mathrm{~m})$ a $24 \mathrm{~ms}$ (30 m) y para Ethernet convencional de 17 $\mathrm{ms}(2 \mathrm{~m})$ a $23 \mathrm{~ms}(30 \mathrm{~m})$.
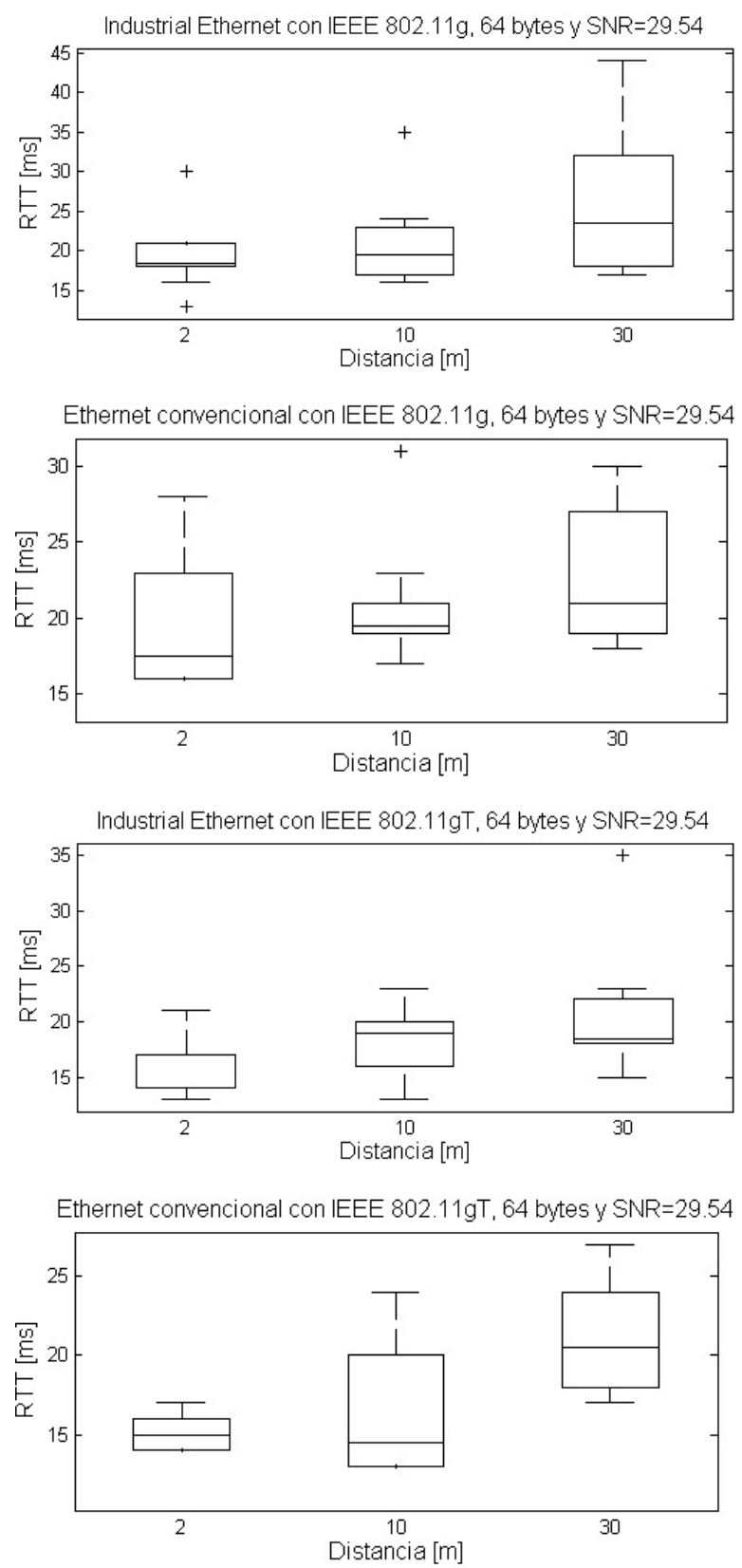

Fig. 8. Resultados obtenidos variando la distancia con $\mathrm{SNR}=29,54 \mathrm{dBm}$. Fuente: Autores 
En este último se obtuvieron menores RTT que para el Industrial Ethernet, excepto en $802.11 \mathrm{gT}$ que muestra una media y una dispersión menor que el convencional. La Fig. 9 presenta los resultados con SNR promedio de 0,66 dBm. Estos evidencian un aumento del RTT medio con respecto a los tomados para SNR de 29,54 $\mathrm{dBm}$.
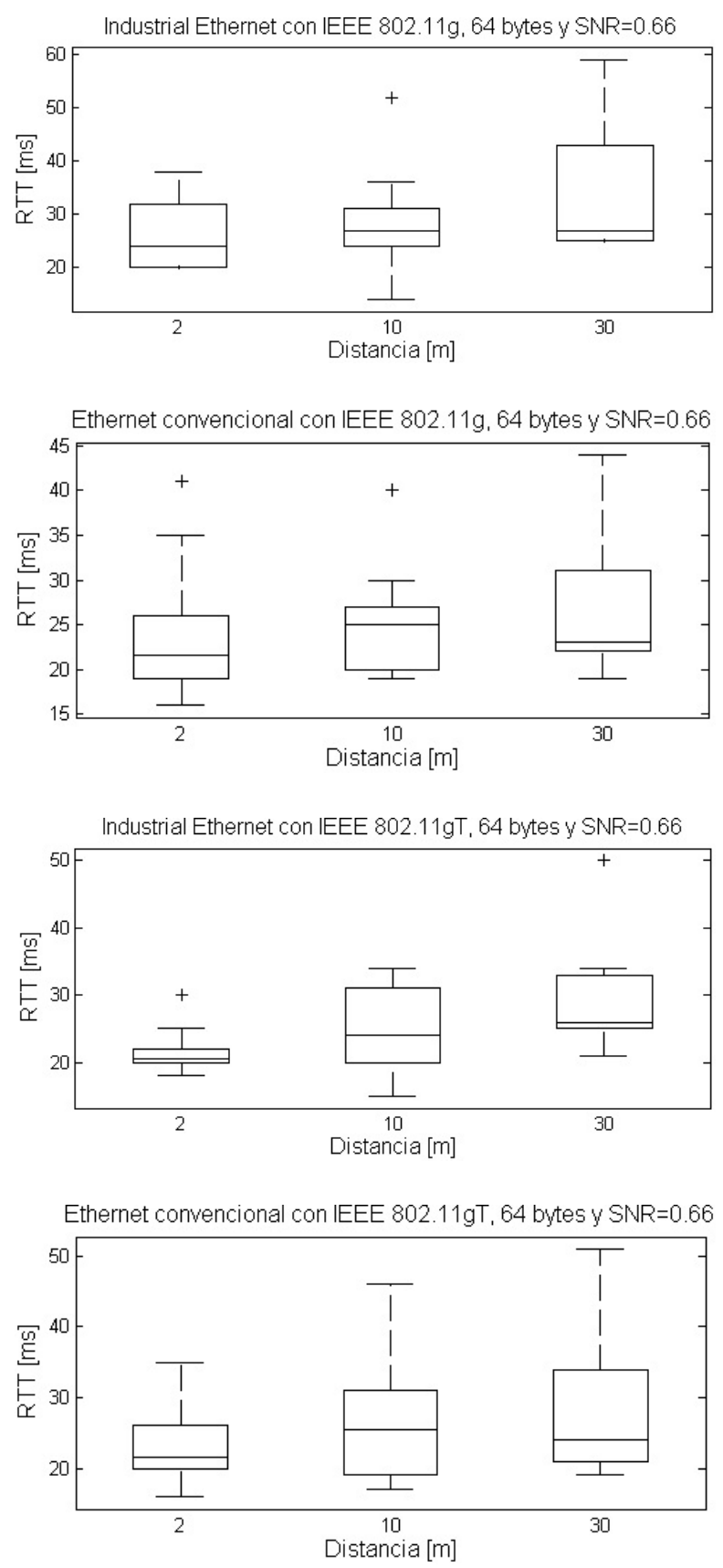

Fig. 9. Resultados obtenidos variando la distancia con $\mathrm{SNR}=0,66 \mathrm{dBm}$. Fuente: Autores
Por ejemplo, se pasó de 18 a $24 \mathrm{~ms}$ con $802.11 \mathrm{~g}$ en Industrial Ethernet y de $17 \mathrm{a}$ $23 \mathrm{~ms}$ en Ethernet convencional con 802.11 g, además se ve un menor RTT en 802.11 gT que en $802.11 \mathrm{~g}$ para ambos casos.

\subsection{PER}

En la Fig. 10 se presentan los resultados obtenidos para el PER en función de SNR. Se observa una gran diferencian entre el Ethernet convencional y el Industrial Ethernet, ya que para el primero el PER va de 29 a $20 \%$, y para el segundo va de 9 a 0,5\% mostrando un mejor resultado el Industrial Ethernet para IEEE 802.11gT.

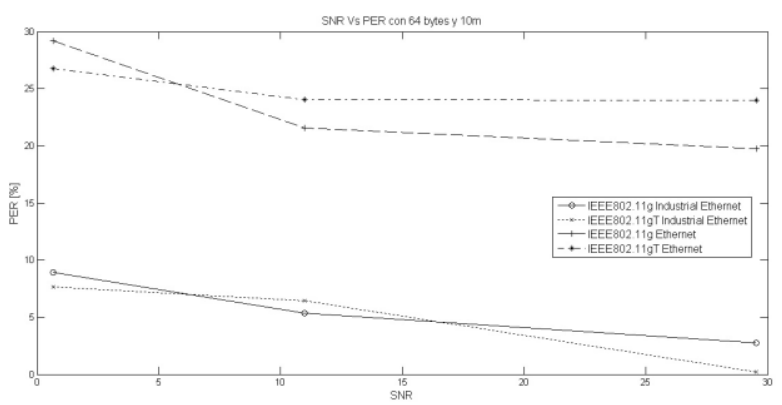

Fig. 10. SNR vs PER con 64 bytes y 10 m. Fuente: Autores

\subsection{Resultados en un proceso de control en tiempo real}

Para el proceso de control en tiempo real, se tomaron ocho medidas con dos SNR para los protocolos 802.11g y gT a una distancia entre los AP de 30 metros. En la Tabla 6 se muestran los promedios de las ocho medidas de tiempo que tarda el motor en llegar al valor deseado una vez se envía el Set Point.

Estos resultados muestran que los promedios aumentan cuando decrece la SNR. Nuevamente, se observa el buen desempeño de Industrial Ethernet con $802.11 \mathrm{gT}$ ante el ruido, que comparado con $802.11 \mathrm{~g}$, tiene un promedio de tiempo $24,38 \mathrm{~ms}$ menor. En la Fig. 11 se observa que la respuesta del protocolo variando el SNR es casi la misma. 
Desempeño de redes inalámbricas y redes industriales inalámbricas en procesos de control en tiempo real...

Tabla 6. Tiempo promedio que tarda el motor en llegar al Set Point desde que se envía su valor. Fuente: Autores

\begin{tabular}{cccc}
\hline & Estándar & SNR [dBm] & Promedio[s] \\
\hline & IEEE $802.11 \mathrm{~g}$ & 30 & 3,44 \\
Industrial & a $30 \mathrm{~m}$ & 0,66 & 3,48 \\
Ethernet & IEEE $802.11 \mathrm{gT}$ & 30 & 3,45 \\
& a $30 \mathrm{~m}$ & 0,66 & 3,46 \\
& IEEE $802.11 \mathrm{~g}$ & 30 & 3,45 \\
Ethernet & a $30 \mathrm{~m}$ & 0,66 & 3,54 \\
Convencional & IEEE $802.11 \mathrm{gT}$ & 30 & 3,45 \\
& a $30 \mathrm{~m}$ & 0,66 & 3,60 \\
\hline
\end{tabular}

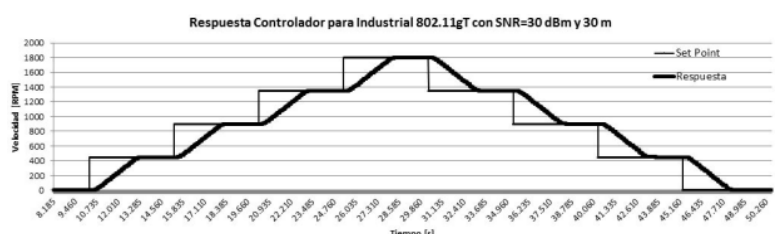

a)

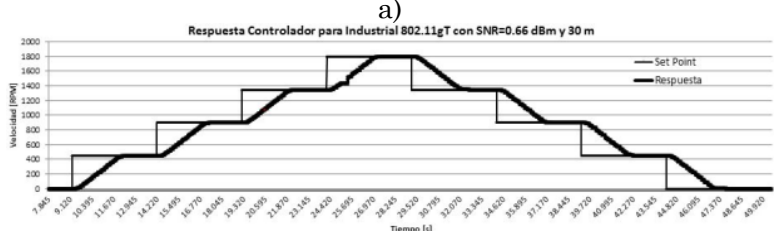

b)

Fig. 11. Respuesta del controlador para Industrial Ethernet IEEE $802.11 \mathrm{gT}$ a $30 \mathrm{~m}$ con a) SNR=30 $\mathrm{dBm}$ y b) $\mathrm{SNR}=0,66$ $\mathrm{dBm}$ b). Fuente: Autores

Caso contrario ocurre con el Ethernet convencional, donde IEEE 802.11gT muestra los peores resultados ante el ruido, con un tiempo $147,5 \mathrm{~ms}$ por encima del que no tiene ruido. En la Fig. 12 se evidencian estos resultados.

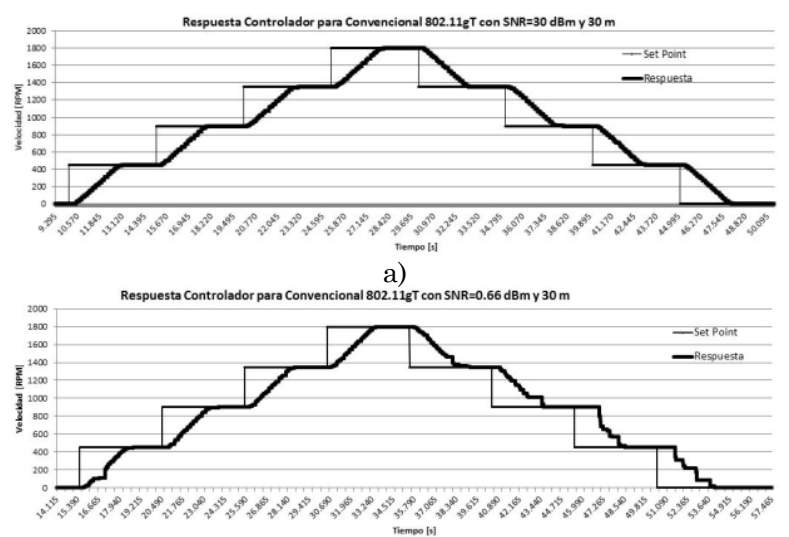

b)

Fig. 12. Respuesta del controlador para Ethernet Convencional IEEE $802.11 \mathrm{gT}$ a $30 \mathrm{~m}$ con a) SNR=30 dBm y b) $\mathrm{SNR}=0,66 \mathrm{dBm}$. Fuente: Autores

\section{CONCLUSIONES}

El uso creciente de las redes Ethernet en el piso de la pirámide de automatización ha hecho que muchas compañías desarrollen dispositivos para operar bajo los requerimientos característicos de este nivel, en la actualidad denominada red Industrial Ethernet. Muchos de estos equipos se venden a muy alto costo, por eso en este artículo se evaluó el desempeño de dos redes inalámbricas, una con dispositivos del Ethernet convencional, y otra con dispositivos de Industrial Ethernet. En primera instancia, analizando las Tablas 4 y 5 se pueden observar algunas diferencias a favor de los AP Scalance de Siemens: un mayor número de protocolos de comunicación que permitiría, de acuerdo con los niveles y frecuencias de interferencia, seleccionar el más adecuado entre un mayor número de posibilidades que el D-Link; menor sensibilidad de recepción $(-96 \mathrm{dBm}$ contra $-92 \mathrm{dBm}$ de D-Link) que permite lograr un mayor alcance en la comunicación, aunque se recomienda instalar los AP lo más cercano posible; y por ultimo Scalance tiene un mayor grado de protección reflejado en mayor temperatura y humedad de operación con rangos desde los -20 a $60 \mathrm{C}^{\circ}$ y $95 \%$, respectivamente.

Respecto al número de datos se puede observar de las Fig. 6 y 7 resultados muy similares entre Industrial Ethernet y el Ethernet convencional, con variaciones promedio en RTT de algunos milisegundos. También es claro que a mayor número de bytes mayor es el RTT. Con el parámetro distancia, de las Fig. 8 y 9, se puede establecer una conclusión similar a la anterior. Para la SNR se puede observar un buen desempeño de ambos equipos, incluso con una SNR de 0,66 dBm, donde el nivel de potencia del ruido en el emisor es casi igual al nivel de potencia de la señal transmitida. 
Observando las Fig. 8 y 9 se ve claramente cómo el RTT aumenta en la medida que aumenta el nivel del ruido, por eso es importante hacer mediciones de nivel de ruido y potencia en la planta para determinar que banda $(2,4$ o $5 \mathrm{GHz})$ y qué protocolo es el más indicado para un buen desempeño. De la Fig. 10 se observa cómo el PER aumenta cuando la SNR disminuye ocasionando mayores retardos en la comunicación. En este aspecto los módulos de Industrial Ethernet muestran un mejor desempeño que los convencionales. En la mayor parte de las pruebas, el protocolo con mejores resultados fue $802.11 \mathrm{gT}$ para Industrial Ethernet, por eso se recomienda establecerlo como protocolo de comunicación para aplicaciones de control en el piso de la pirámide.

Por último, se puede concluir que bajo los parámetros y condiciones evaluados, las redes inalámbricas pueden servir como sistema de comunicación en aplicaciones de control con retardos permisibles de hasta $50 \mathrm{~ms}$, además, los resultados muestran un mejor desempeño de las redes Industrial Ethernet con respecto a las convencionales con diferencias en los RTT de milisegundos. Por lo tanto, se recomienda establecer qué riesgo representa para el proceso a controlar estos retardos y así determinar si los equipos convencionales aplican. Igualmente, se deben tener en cuenta las condiciones de humedad y temperatura ya que pueden afectar el correcto funcionamiento de los dispositivos.

Del análisis de los resultados, se puede sugerir que en aplicaciones donde los retardos no sean críticos para el proceso, y luego de un análisis técnico-económico, es factible utilizar comunicaciones inalámbricas convencionales en lugar de las industriales para disminuir costos sin sacrificar la calidad del servicio. Como trabajo futuro se podría ampliar la investigación teniendo en cuenta parámetros relacionados con el flujo continuo de datos, con una red compuesta de múltiples nodos.

\section{REFERENCIAS}

[1] J. H. Taylor, J. Akerberg, H. M. S. Ibrahim, and M. Gidlund, "Safe and secure wireless networked control systems," in 2012 IEEE International Conference on Control Applications, 2012, pp. 871-878.

[2] B. K. Chejerla and S. Madria, "Securing a Wireless Networked Control System Using Information Fusion," in 2012 IEEE 31st Symposium on Reliable Distributed Systems, 2012, pp. 475-476.

[3] S. X. Ding, P. Zhang, S. Yin, and E. L. Ding, "An Integrated Design Framework of Fault-Tolerant Wireless Networked Control Systems for Industrial Automatic Control Applications," IEEE Trans. Ind. Informatics, vol. 9, no. 1, pp. 462-471, Feb. 2013.

[4] J. Song, A. Mok, D. Chen, and M. Nixon, "Challenges of wireless control in process industry," in Workshop on Research Directions for Security and Networking in Critical Real-Time and Embedded Systems, 2006.

[5] G. W. Irwin, J. Colandairaj, and W. G. Scanlon, "An Overview of Wireless Networks in Control and Monitoring," in Computational Intelligence, vol. 4114, D.-S. Huang, K. Li, and G. W. Irwin, Eds. Berlin, Heidelberg: Springer Berlin Heidelberg, 2006, pp. 1061-1072.

[6] S. Savazzi, S. Guardiano, and U. Spagnolini, "Wireless critical process control in oil and gas refinery plants," in 2012 IEEE International Conference on Industrial Technology, 2012, pp. 1003-1008.

[7] M. Li, P. Zeng, F. Zhang, H. Wang, and R. Zhong, "Structure and protocols of wireless industrial control network," in 2010 International Conference on Computer Application and System Modeling (ICCASM 2010), 2010, pp. V6-140-V6-143.

[8] C. Cheng, P. Hsiao, H. Kung, and D. Vlah, "Performance Measurement of 802.11a Wireless Links from UAV to Ground Nodes with Various Antenna Orientations," in Proceedings of 15th International Conference on Computer Communications and Networks, 2006, pp. 303-308.

[9] M. Collotta, L. Lo Bello, E. Toscano, and O. Mirabella, "Dynamic load balancing techniques for flexible wireless industrial networks," in IECON 2010 - 36th Annual Conference on IEEE Industrial Electronics Society, 2010, pp. 1329-1334.

[10] U. H. S. Asrar and D. A.-M. Yousuf, "On the design of a wireless network in an industrial environment," in 2010 IEEE International Conference on Communication Systems, 2010, pp. 756-760.

[11] K. Kunert, E. Uhlemann, and M. Jonsson, "Enhancing reliability in IEEE 802.11 based real-time networks through transport layer retransmissions," in International Symposium on Industrial Embedded System (SIES), 2010, pp. 146-155.

[12] L. Seno, S. Vitturi, and F. Tramarin, "Influence of real components behavior on the performance of wireless industrial communication systems," in 2011 IEEE International Symposium on Industrial Electronics, 2011, pp. 1224-1229.

[13] C. Rojas and P. Morell, "Guidelines for Industrial Ethernet infrastructure implementation: A control engineer's guide," in 2010 IEEE-IAS/PCA 52nd Cement Industry Technical Conference, 2010, pp. 1-18.

[14] V. Lucan, P. Simacek, J. Seppälä, and H. Koivisto, "Bluetooth and Wireless LAN Applicability for RealTime Control," in Automaatio2003, 2003. 
Desempeño de redes inalámbricas y redes industriales inalámbricas en procesos de control en tiempo real...

[15] M. Islam, M. A. Hannan, S. A. Samad, and A. Hussain, "Bit-Error-Rate (BER) for modulation technique using Software defined Radio," in 2009 International Conference on Electrical Engineering and Informatics, 2009, pp. 445-447.

[16] R. C. Lozoya Gámez, P. Martí, M. Velasco, and J. M.
Fuertes, "Wireless Network Delay Estimation for Time-Sensitive Applications," 2006.

[17] Siemens, "Siemens SCALANCE-W788_Manual." p. $142,2005$.

[18] D-Link Corporation, "Unified Access Point (AP) Administrator's Guide.” p. 183, 2008. 